\title{
Memoria de las I Jornadas de Estudios Urbanos de la Universidad de Costa Rica
}

\author{
Alejandro Bonilla Castro
}

Reseña

Afiliación: Universidad de Costa Rica, Costa Rica. Escuela de Estudios Superiores en Ciencias Sociales EHESS. Francia.

E-mail: alejandro.bonillacastro@ucr.ac.cr

Recibido: 8 de enero del 2019

Aceptado: 29 de abril del 2020

\section{Alejandro Bonilla Castro}

Máster en Historia por la UCR y docente de la Escuela de Historia de esta institución. Estudiante de doctorado de la Escuela de Altos Estudios en Ciencias Sociales de París (EHESS) y miembro del laboratorio UMR 8504 Géographie-Cités (CNRS). Investigador de la historia de la planificación urbana con enfoque global.

\section{Resumen}

Los problemas urbanos que actualmente afronta la Gran Área Metropolitana (GAM) de Costa Rica son producto de trayectorias históricas que han fortalecido las desigualdades espaciales de su población en ámbitos como el acceso a la vivienda, las opciones de transporte, la seguridad, el trabajo y el ocio. Aunque los estudios urbanos costarricenses han sido prolíficos a lo largo de los años, su también característica dispersión ha dificultado realizar el análisis de los factores políticos, sociales, culturales y económicos que han condicionado el desarrollo urbano de la GAM en el largo plazo y sus efectos en las zonas rurales del país. De ahí que la convocatoria a las I Jornadas de Estudios Urbanos de la Universidad de Costa Rica se realizó con el fin de acercar a los investigadores, colectivos urbanos y los encargados de tomar de decisiones a debatir sobre los enfoques que caracterizan la actual corriente de estudios urbanos. El presente ensayo tiene como propósito recopilar los resultados de las distintas mesas de discusión y actividades complementarias que se realizaron entre el 17 y el 21 de junio del 2019, así como proponer algunas guías que lleven a la realización de futuras actividades de esta índole y el establecimiento de una red de estudios urbanos a nivel centroamericano.

Palabras clave: Costa Rica; eventos; estudios urbanos; planificación urbana.

\section{Dossier of the First Workshop on Urban Studies of the University of Costa Rica}

\section{Abstract}

The urban problems that currently face the Greater Metropolitan Area (GAM) of Costa Rica are the product of historical trajectories that have strengthened the spatial inequalities of its population in areas such as access to housing, transport options, security, work and leisure. Although Costa Rican urban studies have been prolific over the years, their characteristic dispersion has made it difficult to analyze the political, social, cultural and economic dynamics that have conditioned the urban development of GAM in the long term forgetting its effects, as well, in rural areas of the country. Hence, the call for the First Workshop on Urban Studies of the University of Costa Rica was held in order to bring researchers, urban groups and decision-makers close together to discuss the perspectives and approaches that characterize the actual current of urban studies. The purpose of this essay is to summarize the results of the various discussion tables and complementary activities that took place between 17 and 21 June 2019, as well as to propose some guidelines that could lead to the realization of future activities like this and the establishment of a network of urban studies in Central America.

Keywords: Costa Rica; events; urban studies; urban planning. 


\section{Estado de la problemática y sustento de las I Jornadas de Estudios Urbanos}

na de las razones que motivaron la convocatoria a las I Jornadas de Estudios Urbanos es la tensión que existe dentro de la GAM entre los modelos urbanos heredados del siglo XX y otros que, con la llegada de la presente centuria, han modificado la forma de vivir, percibir y ordenar las ciudades. Algunas de las manifestaciones de estos "nuevos" modelos han sido la aparición de torres de apartamentos, de alternativas al transporte público tradicional (buses y taxis), la gentrificación de varios barrios capitalinos y la aparición de circuitos de turismo cultural que se apoyan en los vestigios de la ciudad bucólica y su nostalgia. Al mismo tiempo, estas prácticas refuerzan las desigualdades espaciales de otros sectores de la GAM debido a su ausencia (¿para bien o para mal?) que se materializan en espacios urbanos deprimidos, la inseguridad ciudadana y las largas horas de desplazamiento.

Por otro lado, están los proyectos de infraestructura y de planificación en transportes que han tomado los titulares noticiosos en los últimos años, siendo los más sonados los de sectorización y el tren interurbano. Esto se debe, entre otros factores, a su uso político por el actual gobierno del presidente Carlos Alvarado Quesada y el papel que asumió en esta estrategia la Arquitecta Claudia Dobles Camargo, primera dama. La presencia recurrente de estos temas en la opinión pública ha favorecido su creciente debate en sectores de la academia y la ciudadanía, a fin de comprender las complejidades, aspiraciones, conceptos y visiones del fenómeno urbano.

Con relación a la academia, esta se ha caracterizado por haber producido numerosos trabajos e investigaciones. En las décadas de 1950 y 1960 varios trabajos se abocaron por definir los objetivos del urbanismo, la zonificación, la economía planificada, la vivienda social y su rol dentro del recién fundado Instituto Nacional de Vivienda y Urbanismo (INVU) en 1954 (Carazo, 1958; Vargas, 1958; Jenkins y Crespo, 1958; Gordienko, 1955; Arguedas, 1960).

En la década de 1970 aparecieron trabajos más críticos hacia el fenómeno de la metropolización, los desequilibrios del modelo desarrollista, el clientelismo en los proyectos de vivienda (Morales, 1976; Montoya, 1976), y durante la crisis de los ochenta recibieron tratamiento la imagen urbana, los movimientos sociales y el crecimiento urbano y sus efectos a nivel regional (Argüello, 1980; Sánchez. et al, 1982; Rodríguez, et al, 1985; Jiménez, 1985; Brenes y Zúñiga, 1985; Altezor, 1986; Woodbridge, 1988; Hernández, et al., 1984; Hall, 1983).

En los noventa y los dos primeros decenios del siglo XXI, las perspectivas de análisis privilegiaron los análisis culturales y el desarrollo urbano de la ciudad capital (Palmer, 1996; Fumero, 1996; Quesada, 2004 y 2011), la cuestión social urbana (Elizondo, 2009; Botey, 2013; Malavassi, 2014; Izquierdo, 2016), la apropiación del espacio público (Sánchez, et al., 2012; Durán, 2013), la segregación urbana (Van Lidth de Jeude y Shütte, 2010; Alvarado y Jiménez, 2014; Jiménez, 2017), la movilidad urbana (Jiménez, 2018), los modelos urbanos (Martínez, 2009), la gentrificación (Molina, 2019), la resiliencia urbana (Picado, 2019; Girot, 2019), la historia global (Bonilla, 2018) y el derecho a la ciudad (Jouffe, 2015).

Lo que deseo destacar con este brevísimo - y para nada completo - estado de la cuestión es que los estudios urbanos en Costa Rica han ido ampliando su espectro analítico al pasar de las descripciones del cambio de la trama urbana, a preocuparse por la amplia gama de variables económicas, sociales, políticas y culturales que explican esas variaciones. No obstante, como lo señalé atrás, la gran mayoría de estas investigaciones se encuentran dispersas y a la fecha no hay un repositorio especializado en temas urbanos. También, si miramos hacia la región centroamericana, la situación es aún más complicada, ya que a pesar de que se encuentran los valiosos trabajos de Mario Lungo (2004, 2015), Daniela Navarrete (2012 y 2018) y algunas investigaciones de FLACSO (Pérez y Pujol, 2003; Séguin y Negrón, 2006), la tarea de realizar análisis en perspectiva comparada (sincrónica o diacrónica) no deja de estar ajena a serias complicaciones, entre ellas, el acceso a las fuentes documentales y textos producidos en el área. 
Ciertamente estas preocupaciones guiaron la intención de proponer un espacio interinstitucional de diálogo entre quienes en la actualidad realizan investigaciones en el campo de los estudios urbanos, con el objetivo de que se sigan celebrando de forma frecuente jornadas y actividades complementarias que vayan sumando nuevas interrogantes, nuevas metodologías de estudio y procesos de intercambio de saberes entre investigadores costarricenses y aquellos de la región centroamericana.

\section{Organización de las I Jornadas de Estudios Urbanos}

\section{La planificación de las mesas}

Tabla 1. Ponentes y moderadores de las mesas de discusión y conversatorio de las I Jornadas de Estudios Urbanos de la Universidad de Costa Rica (20 de junio 2019).

Fuente: Programa I Jornada de Estudios Urbanos de la Universidad de Costa Rica (19 y 20 de junio del 2019).

Tabla 2. Agenda complementaria de las Jornadas de Estudios Urbanos de la Universidad de Costa Rica (17-21 de junio 2019).

Fuente: Programa I Jornadas de Estudios Urbanos de la Universidad de Costa Rica.
Las I Jornadas de Estudios Urbanos de la UCR celebradas el 20 de junio del 2019 en el Auditorio de la Facultad de Educación estuvieron compuestas por tres mesas de discusión y un conversatorio de cierre (Tabla 1). Las actividades complementarias, se detallan en la Tabla 2.

\begin{tabular}{|c|c|c|c|c|}
\hline & $\begin{array}{l}\text { Mesa Metodologías } \\
\text { en Estudios Urbanos }\end{array}$ & Mesa Movilidad Urbana & $\begin{array}{l}\text { Mesa Desigualdades } \\
\text { socioespaciales y } \\
\text { ordenamiento territorial }\end{array}$ & $\begin{array}{l}\text { Retos y oportunidades } \\
\text { de la planificación } \\
\text { urbana en Costa Rica }\end{array}$ \\
\hline \multirow[t]{5}{*}{ Ponentes } & $\begin{array}{l}\text { Dra. } \quad \text { Daniela } \\
\text { Navarrete C. }\end{array}$ & Licda. Carla Quesada A. & Licda. María J. Guillén A. & Dra. Zuhra Sasa M. \\
\hline & $\begin{array}{l}\text { M.Sc. Clara Quintilla } \\
\text { P. }\end{array}$ & M.Sc. Roy Allan Jiménez & M.Sc. Wendy Molina V. & Arq. Tomás Martínez B. \\
\hline & $\begin{array}{l}\text { M.Sc. } \quad \text { Alejandro } \\
\text { Bonilla C. }\end{array}$ & $\begin{array}{l}\text { M.Sc. Gustavo Jiménez } \\
\text { B. }\end{array}$ & Lic. Andrés Jiménez C. & Arq. David Porras A. \\
\hline & $\begin{array}{l}\text { Bach. Ismayaira } \\
\text { Guillèn M. }\end{array}$ & M.Sc. Pascal Girot P.* & & $\begin{array}{l}\text { Dra. Daniela Navarrete } \\
\text { C. }\end{array}$ \\
\hline & $\begin{array}{l}\text { Dra. Rosa Malavassi } \\
\text { A. }\end{array}$ & & & \\
\hline $\begin{array}{l}\text { Moderadores y } \\
\text { comentaristas de } \\
\text { mesa }\end{array}$ & Dr. Anthony Goebel M. & M.Sc. Luis Durán S. & $\begin{array}{l}\text { Dr. Víctor Delgadillo P. y } \\
\text { M.Sc. Adriana Sánchez L. }\end{array}$ & Dr. Sergio Villena F. \\
\hline
\end{tabular}

* No asistió a las Jornadas por otros compromisos universitarios.

\begin{tabular}{|c|c|c|c|}
\hline Nombre actividad & Lugar & Fecha & Participantes \\
\hline $\begin{array}{l}\text { Conversatorio. El estudio } \\
\text { de la gentrificación en } \\
\text { Latinoamérica. }\end{array}$ & Sala de audiovisuales (IIS) & 17 de junio 2019, 1:00 pm & Dr. Víctor Delgadillo P. \\
\hline $\begin{array}{l}\text { Taller. El deterioro y la } \\
\text { regeneración urbana. } \\
\text { Metodologías para el análisis } \\
\text { con perspectiva histórica en } \\
\text { Centroamérica. }\end{array}$ & $\begin{array}{l}\text { CEDOCIHAC (Facultad de } \\
\text { Ciencias Sociales) }\end{array}$ & 17 de junio 2019, 3:00 pm & Dra. Daniela Navarrete C. \\
\hline $\begin{array}{l}\text { Reunión de investigadores } \\
\text { de las I Jornadas de Estudios } \\
\text { Urbanos. }\end{array}$ & $\begin{array}{l}\text { CIHAC (Facultad de Ciencias } \\
\text { Sociales) }\end{array}$ & 18 de junio 2019, 9:00 am & $\begin{array}{l}\text { Dr. Víctor Delgadillo, Dra } \\
\text { Daniela Navarrete, Dr. Anthony } \\
\text { Goebel, Licda. María J. Guillén, } \\
\text { Lic. Andrés Jiménez, M.Sc. } \\
\text { Wendy Molina, Ing. Jessie } \\
\text { Vega, M.Sc. Gustavo Jiménez, } \\
\text { M.Sc. Alejandro Bonilla, Bach. } \\
\text { Ismayaira Guillén. }\end{array}$ \\
\hline $\begin{array}{l}\text { Charla. Laboratorios urbanos: } \\
\text { modelos de ciudad en la } \\
\text { Tegucigalpa liberal y neoliberal } \\
\text { (S. XIX y XX) }\end{array}$ & Sala de audiovisuales (IIS) & 18 de junio 2019, 2:00 pm & $\begin{array}{l}\text { Dra. Daniela Navarrete C. } \\
\text { M.Sc. Alejandro Bonilla C. }\end{array}$ \\
\hline $\begin{array}{l}\text { Charla. Metáforas de las } \\
\text { ciudades latinoamericanas. }\end{array}$ & $\begin{array}{l}\text { Auditorio de la Escuela de } \\
\text { Arquitectura. }\end{array}$ & 18 de junio 2019, 6:00 pm & $\begin{array}{l}\text { Dr. Víctor Delgadillo P. } \\
\text { Dra. Zuhra Sasa M. } \\
\text { Dra. María del Carmen Araya. } \\
\text { Dra. Helga von Breymann }\end{array}$ \\
\hline $\begin{array}{l}\text { Entrevista con los invitados } \\
\text { internacionales. }^{1}\end{array}$ & $\begin{array}{l}\text { Oficina de Divulgación e } \\
\text { Información }\end{array}$ & 19 de junio 2019 & $\begin{array}{l}\text { Dra. Daniela Navarrete C. } \\
\text { Dr. Víctor Delgadillo P. } \\
\text { M.Sc. Luis Durán S. } \\
\text { Francisco Ruiz León. }\end{array}$ \\
\hline $\begin{array}{l}\text { Mesa inaugural. Tren Rápido } \\
\text { de Pasajeros. ¿Una alternativa } \\
\text { para mejorar la movilidad } \\
\text { urbana de la GAM? }\end{array}$ & $\begin{array}{l}\text { Auditorio de la Escuela de } \\
\text { Arquitectura. }\end{array}$ & 19 de junio 2019, 6:00 pm & $\begin{array}{l}\text { Dra. Ana M. Botey S. } \\
\text { Ing. Olman Vargas Z. } \\
\text { M.Sc. Gustavo Jiménez B. } \\
\text { M.Sc. Marcela Otárola G. }\end{array}$ \\
\hline $\begin{array}{l}\text { Reunión con estudiantes con } \\
\text { investigaciones en estudios } \\
\text { urbanos }\end{array}$ & $\begin{array}{l}\text { CIHAC (Facultad de Ciencias } \\
\text { Sociales) }\end{array}$ & 21 de junio 2019, 9:00 am & $\begin{array}{l}\text { Dra. Daniela Navarrete C. } \\
\text { Dr. Victor Delgadillo P. } \\
\text { Estudiantes convocados. }\end{array}$ \\
\hline $\begin{array}{l}\text { Cineforo: Desigualdades } \\
\text { socioespaciales en la ciudad } \\
\text { neoliberal. }\end{array}$ & $\begin{array}{l}\text { Aula 204, Facultad de Ciencias } \\
\text { Sociales. }\end{array}$ & 21 de junio 2019, 2:00 pm & $\begin{array}{l}\text { Dra. Daniela Navarrete C. } \\
\text { Dr. Víctor Delgadillo P. } \\
\text { M.Sc. Alejandro Alvarado A. }\end{array}$ \\
\hline
\end{tabular}


Aunque durante la planificación de las Jornadas se previeron más espacios de discusión, factores como la disponibilidad de tiempo y espacio en el campus obligaron a unificar varias temáticas en una sola mesa. Un ejemplo de ello fue la de Desigualdades socioespaciales y ordenamiento territorial, que surgió de la fusión de cuatro mesas planificadas al inicio. Otro reto sustancial fue mantener la paridad de género en cada una de ellas. Solamente la de Movilidad Urbana fue la excepción, pues contó sólo con una participante a pesar de que se enviaron varias invitaciones a mujeres especialistas en la materia, pero declinaron la invitación por diversos motivos.

También se procuró mantener la representatividad de los centros organizadores en cada una de las actividades de las Jornadas. Así, además del auditorio de la Facultad de Educación, se realizaron actividades en el Centro de Investigaciones Históricas de América Central (CIHAC) y en su Centro de Documentación (CEDOCIHAC), la Facultad de Ciencias Sociales (Aula 204), el auditorio de la Escuela de Arquitectura y la Sala de Audiovisuales del Instituto de Investigaciones Sociales (IIS). Asimismo, el Centro de Estudios Mexicanos (CEM-UNAM) fue anfitrión de un convivio entre varios investigadores participantes y un grupo de estudiantes de la Universidad Nacional Autónoma de México (UNAM) quienes se integraron a las actividades de las Jornadas.

\section{La imagen de las Jornadas}

Para la imagen de las jornadas se escogió la perspectiva del proyecto para el Centro Cívico de San José, elaborado por el INVU a mediados de la década de 1950. Se consideró que, al poner en valor la investigación actual en estudios urbanos, también se debía hacer lo mismo con los primeros urbanistas costarricenses quienes se plantearon dotar a la capital de lo que consideraban, en aquel entonces, de las soluciones punteras en cuanto a ordenamiento urbano. El Centro Cívico simboliza ese esfuerzo profesional. Las redes internacionales jugaron un papel relevante en la creación del urbanismo como práctica profesional en Costa Rica y las imágenes de modernidad que se deseaban aplicar en San José. (Imagen 1). La estudiante Adriana Salvador Luna, asistente del Laboratorio Ciudad y Territorio (LACITE) de la UCR, fue la encargada de elaborar el diseño e imagen del afiche principal de las Jornadas.

\section{La colaboración internacional}

Además de la presencia de instituciones de educación superior nacionales (UCR, UNA y TEC), el gobierno central (Ministerio de Obras Públicas y Transportes) y las instituciones autónomas (INVU), tuvieron representatividad varios centros académicos internacionales. Por medio de la Oficina de Asuntos Internacionales y Cooperación Externa (OAICE) de la UCR, fue posible traer a dos especialistas en temas urbanos como invitados especiales de las I Jornadas de Estudios Urbanos: el Dr. Víctor Delgadillo Polanco, de la UNAM y la Dra. Daniela Navarrete Cálix de la Escuela Agrícola Panamericana Zamorano de Honduras. En el caso del Dr. Delgadillo se contó con la estrecha colaboración del Dr. Carlos Valdés González y el Mtro. Héctor Perdomo Velázquez del CEM-UNAM, quienes facilitaron la visita del académico mexicano en conjunto con la Dra. Zuhra Sasa Marín, Directora de la Escuela de Arquitectura. En cuanto a la Dra. Navarrete, el apoyo logístico se obtuvo de la OAICE y de la Dra. Ana María Botey Sobrado, Directora de la Escuela de Historia. La Dra. Navarrete y la M.Sc. en Antropología Clara Quintilla Piñol fueron además representantes de la Escuela de Altos Estudios en Ciencias Sociales de París (EHESS).

\section{Resultados de las I Jornadas de Estudios Urbanos}

\section{Síntesis de las problemáticas analizadas}

El problema de la movilidad urbana fue una de las ideas centrales que impulsaron la convocatoria a las I Jornadas de Estudios Urbanos, a raíz de la discusión del 
Imagen 1. Afiche de las IJEU y el anteproyecto del Centro Cívico para la ciudad de San José de 1955.

Fuente: Adriana Salvador Luna, Gustavo Jiménez Barboza y Alejandro Bonilla Castro. proyecto del Tren Rápido de Pasajeros (TRP) y la mala experiencia ciudadana en sus desplazamientos en la GAM. En relación con el actual proyecto del INCOFER y el Despacho de la Primera Dama, uno de los aspectos medulares que debe llamar nuestra atención es la trayectoria histórica que tuvo el ferrocarril en Costa Rica que, hasta la década de 1980, se le consideró como un servicio público regional, regulado y promotor del desarrollo general. ¿Podemos hablar en los mismos términos con el TRP?

- CONTARIDAD DE

\section{JORNADAS}

DE ESTUDIOS URBANOS

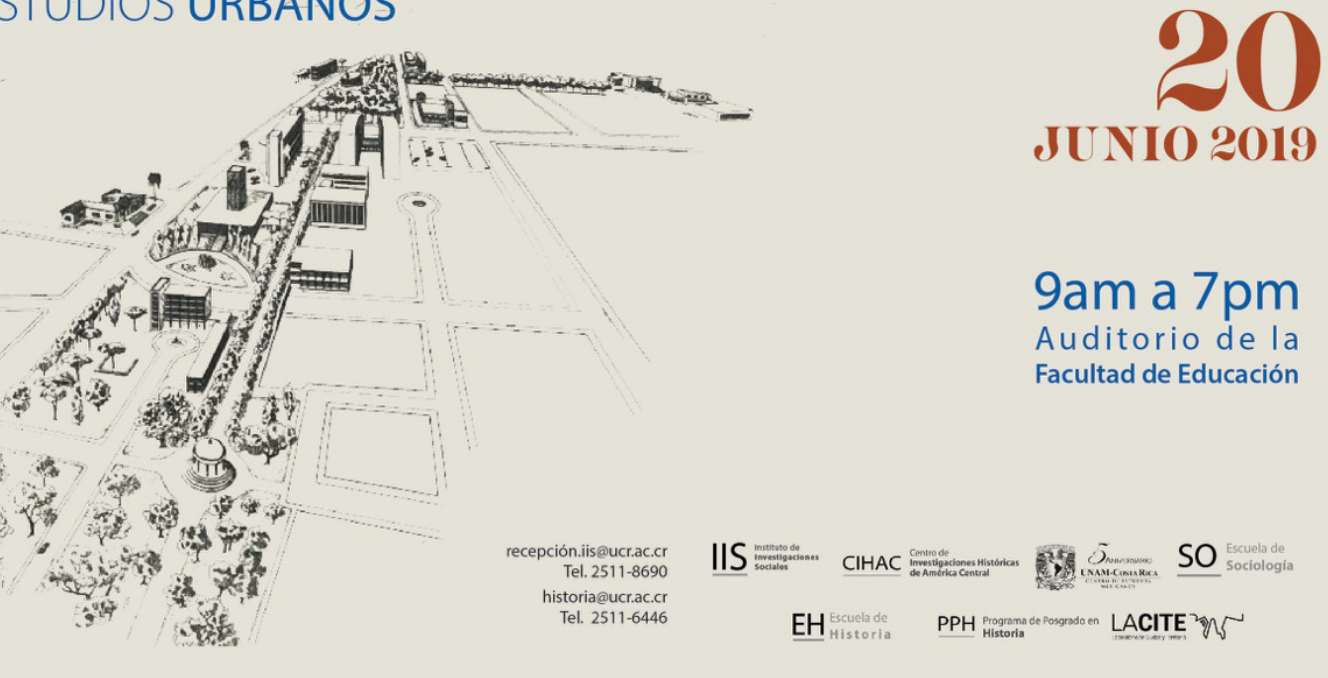

La mesa inagural de las Jornadas concluyó que la modernización del servicio de ferrocarriles aún se encuentra lejos de esta meta. Primeramente, debido al limitado alcance geográfico del proyecto dentro de la GAM, ya que se sustentaría en el mismo trazado realizado en el siglo XIX y que excluye espacialmente a aquellos habitantes que, debido a la expansión desordenada de la GAM de las últimas décadas, viven a las afueras de los centros urbanos por donde transitaría el moderno ferrocarril.

En segunda instancia, la solución a los problemas de desplazamiento no depende únicamente del tren, sino que es necesaria la vinculación con otras formas de movilidad. Tal es el caso del proyecto de sectorización del transporte de autobuses impulsado desde el MOPT y expuesto por el Máster Roy-Allan Jiménez que pretende, a partir de los llamados "nodos", integrar habitabilidad, espacio público e infraestructura complementaria al transporte público. A partir de la idea central de que la movilidad "inicia desde que se sale de casa", el confort y la eficacia del transporte se vincula con la vivencia del espacio urbano circundante. Tales ideas se complejizan, cuando se toman en cuenta las tácticas de desplazamiento de los ciudadanos, la apropiación del espacio e incluso el estatus socioeconómico que los condiciona a elegir el medio de transporte, según advertía el Máster Gustavo Jiménez.

Además, la Lic. Carla Quesada del Centro para la Sostenibilidad Urbana (CPSU) recalcó que el transporte público es usado eminentemente por las mujeres debido a su "doble jornada laboral", es decir, la combinación de su rol como profesional y de "cuido de los (as) otros (as)". Se suma a esto la inseguridad que experimentan las mujeres al ser víctimas del acoso callejero y su condición socioeconómica, producto de la brecha salarial respecto a los hombres. La integralidad de ese "confort" y la "eficacia" del transporte público y la infraestructura complementaria, pueden cuestionarse a partir de la perspectiva de género, pues las mujeres elegirían sus rutas de desplazamiento, se apropiarían del espacio público y usarían el transporte público si estos incorporaran mayores y mejores garantías para su seguridad y sus derechos laborales. Con base en esto, cabe destacar que la desigualdad espacial incorpora numerosas variables que deben tomarse en cuenta para lograr una mejor democratización del espacio urbano, por lo que el diálogo interdisciplinario debe ser una constante.

Sin embargo, esta desigualdad no es un fenómeno que se restringe únicamente a las ciudades. La Lic. María José Guillén recalcó precisamente que una deuda de las ciencias sociales - y con ello de los estudios urbanos - es eliminar la dicotomía entre 
lo urbano y lo rural a la hora de analizar los procesos de construcción del territorio y el espacio. La ciudad de Palmar Sur se utilizó como estudio de caso para mostrar cómo la influencia de la Compañía Bananera durante cincuenta años eliminó los vestigios de otras formas de vida (como la indígena y la campesina), alteró la sociabilidad de sus habitantes y organizó el territorio circundante mediante jerarquías impuestas, provocando una ruptura socioespacial. Pero estas compañías transnacionales no pueden actuar sin la complacencia del poder local o nacional que, por medio de su institucionalidad, promociona agendas de "diversificación productiva", o la explotación del paisaje rural como mercancía, las cuales serían ecos de un modelo neoliberal que promueve fundamentalmente el consumo, por sobre otras realidades humanas, en las ciudades.

El rol del Estado como promotor de un modelo neoliberal es esencial en ambos casos, tema en el que profundizó el Lic. Andrés Jiménez con su análisis del Programa de Regeneración y Repoblamiento de San Joséy los proyectos urbanos que trae aparejados, como el de la Ciudad Tecnológica. En ellos, advirtió Jiménez, hay una tendencia dentro de la Municipalidad de San José a ver estas iniciativas de ordenamiento territorial como "rescates" de zonas pauperizadas de la capital mediante la explotación inmobiliaria del espacio, su comercialización y la atracción de una mano de obra especializada con alto poder adquisitivo, a costa de la paulatina expulsión de la población original y el lucro inmobiliario. Es decir, se revelan como iniciativas de gentrificación urbana.

Sin embargo, este fenómeno no es exclusivo de las ciudades. La M.Sc. Wendy Molina demostró cómo, también en las zonas rurales, los procesos de captación de la renta por parte de las inmobiliarias son responsables de expulsar a las familias de sus tierras en las zonas limítrofes de la GAM o más allá de ella, lo que demuestra la interrelación de estos procesos y la necesidad de analizarlos de forma sincrónica. Respecto a la gentrificación, vale la pena recuperar la reflexión del Dr. Delgadillo y la Dra. Navarrete sobre este concepto, su historicidad y sus alcances analíticos, es decir, ¿conviene utilizar este concepto para analizar las dinámicas inmobiliarias y sus efectos socioeconómicos en Costa Rica? ¿Es un concepto que debemos considerarse a priori peyorativo? Una pista que invita a reflexionar sobre esto último es el concepto de "gentrificación convival", nombre que recibe una "estrategia de supervivencia" aplicada por vecinos de algunos barrios madrileños para evitar la pauperización de su entorno, caso visto durante el cineforo "Desigualdades socioespaciales de la ciudad neoliberal." Asimismo, es preciso profundizar en el rol de los grupos inmobiliarios en este proceso.

A nivel metodológico, la propuesta de investigación de la Bach. Ismayaira Guillén considera precisamente el papel de estos actores empresariales en cuyo caso, la reconstrucción de sus redes ofrece claridad respecto a sus estrategias comerciales y la lectura que hacen estas del contexto económico y fiscal, así como de las ventajas que obtienen en los recintos de poder local o nacional. Al centrar el análisis en los actores (sean estos dominantes y subalternos) los actuales estudios urbanos costarricenses se alejan cada vez más de ser solo radiografías de las tipologías urbanas y se acercan a las tendencias (de)constructivistas del proceso urbano. La ponencia de la Dra. Rosa Malavassi demostró el dinamismo de la categoría del "patrimonio" si se le considera como el producto de la apropiación, o no, por parte de los actores de las representaciones sociales que definen si un espacio, o un bien, es patrimonio. A similar conclusión llegó la Dra. Navarrete sobre el rol de la memoria colectiva a la hora de planificar la ciudad y las decisiones que existen para que en ciertos espacios se conserven, o no, con el transcurrir del tiempo.

Este enfoque (de)constructivista del espacio urbano y sus representaciones sociales es recuperado también por la Máster Quintilla con el caso de los kibutz urbanos en Israel, portavoces de un movimiento transnacional de comunidades intencionales que se proponen cambiar su entorno vivencial a partir de la experiencia comunitaria, la educación y la cooperación económica. Estos valores, con el paso del tiempo, se arraigan al espacio urbano en cuestión, y su supervivencia depende de la participación democrática de sus miembros y su apropiación por parte de las generaciones subsiguientes. Un indicio de la existencia de este tipo de colectividades se encuentra al sur de Costa Rica con el pueblo de Longo Maï, lo que invita a analizar la extensión de este movimiento transnacional de "comunidades intencionales", sus tipologías y 
tácticas para extender y defender sus formas de convivencia y apropiación de sus espacios urbanos y rurales. Las comunidades intencionales son otra categoría que pueden facilitar el derrumbamiento de la dicotomía de lo "urbano" versus lo "rural" y se inserta en la tendencia marcada por la Lic. Guillén y la M.Sc. Molina.

Finalmente, la ponencia del suscrito, precisamente buscó invitar a leer con el prisma de la historia global, cómo han circulado las ideas de la planificación urbana, traspasando las fronteras del Estado-Nación. Con esta perspectiva, los modelos urbanos y las prácticas que los acompañan o dan fundamento son construcciones históricas multiescala, en donde lo local, lo nacional, lo regional y global están interrelacionados. Con este lente, podemos diseccionar la mecánica interna que constituye y legitima un "modelo urbano", por qué y cómo se eligen las buenas prácticas que lo acompañan, su nivel de apropiación y las resistencias que se generan a la hora de ponerlos en ejecución. Las "metáforas de lo urbano", como las llama el Dr. Delgadillo a esta serie de modelos como "ciudades creativas", "ciudades compactas", "ciudades inteligentes", "ciudades globales" circulan a nivel transfronterizo y se promocionan en espacios privilegiados donde convergen tanto actores políticos, académicos, empresariales, entre otros. La perspectiva global abre numerosas posibilidades de interpretación del hecho urbano y rural, ampliando ciertamente, el rol de los actores sociales que entran en juego dentro del proceso.

Con este escenario, las Jornadas se proyectaron como un espacio de actualización valioso de los estudios urbanos costarricenses, con el fin de insertarlos en las tendencias de investigación actuales a nivel mundial y favorecer, de esta forma, el intercambio de conocimientos con otras latitudes.

\section{Proyección social de las Jornadas}

Las I Jornadas de Estudios Urbanos propuso, además, otros espacios de diálogo entre académicos, funcionarios públicos, miembros de colectivos urbanos, estudiantes y público en general. La mesa de clausura fue un éxito en este sentido al incentivar el diálogo entre las Escuelas de Arquitectura tanto del TEC, como de la UCR con el INVU y establecer propuestas para colaboraciones cercanas entre estas instituciones a nivel de política urbana. Lamentablemente, no fue posible realizarlo durante la mesa inaugural, ya que la participación de la Arq. Claudia Dobles y la presidenta del Instituto Costarricense de Ferrocarriles (INCOFER), la Licda. Elizabeth Briceño Jiménez, no se pudo concretar. Sin duda, el diálogo entre la academia y los los encargados de tomar de decisiones políticas es una tarea pendiente, especialmente cuando estos se encuentran en sus primeras etapas de formulación.

Una de las conclusiones a las que se llegó durante la reunión de investigadores de las Jornadas de Estudios Urbanos, realizada el martes 18 de junio (Imagen 2), fue que la continuidad de este proyecto académico debe promover la combinación de las mesas de discusión con el uso del espacio urbano de las ciudades costarricenses, la incorporación de actividades pedagógicas y especialistas en la educación, la proyección de la Acción Social universitaria en este marco mediante los TCU, los proyectos de extensión docente y cultural, así como llevar a cabo reuniones periódicas entre los especialistas con el fin de discutir, y debatir los resultados de las investigaciones en estudios urbanos.

Por otro lado, tomó realce una propuesta de agenda investigativa en estudios urbanos que incluiría problemáticas como: la construcción histórica del ambiente y el paisaje urbano, las estructuras de financiamiento y redes de poder empresariales, la crítica de las políticas urbanas actuales, el rol de la universidad en la producción del espacio y el territorio, la construcción histórica del patrimonio cultural, la relación Estado organismos internacionales, la transnacionalización de los modelos urbanos, los movimientos sociales urbano-rurales, y promover, finalmente, los análisis comparativos entre casos centroamericanos.

Además, dicha agenda debe venir acompañada de la creación de repositorios en donde se puedan publicar periódicamente los avances de investigación o artículos, compartir fuentes y contactos, para que poco a poco reduzcan la dispersión de la información. La 
Imagen 2. Reunión de investigadores de las Jornadas de Estudios Urbanos. 18 de junio del 2019.

Fuente: Alejandro Bonilla C., 2019. colaboración entre universidades públicas, el gobierno y los colectivos urbanos debe ser más estrecha para poder conseguir estos objetivos. El primer paso hacia esta meta debe ser, a mi juicio, sostener los encuentros regulares entre investigadores vinculados o (as) a los estudios urbanos de forma que se siga fortaleciendo paulatinamente un grupo de trabajo y con ello, se incorporen más agendas de investigación lo que requiere un mayor diálogo entre las unidades académicas y centros de investigación de la UCR, para pasar al siguiente nivel con los nexos entre otras instituciones y colectivos ciudadanos

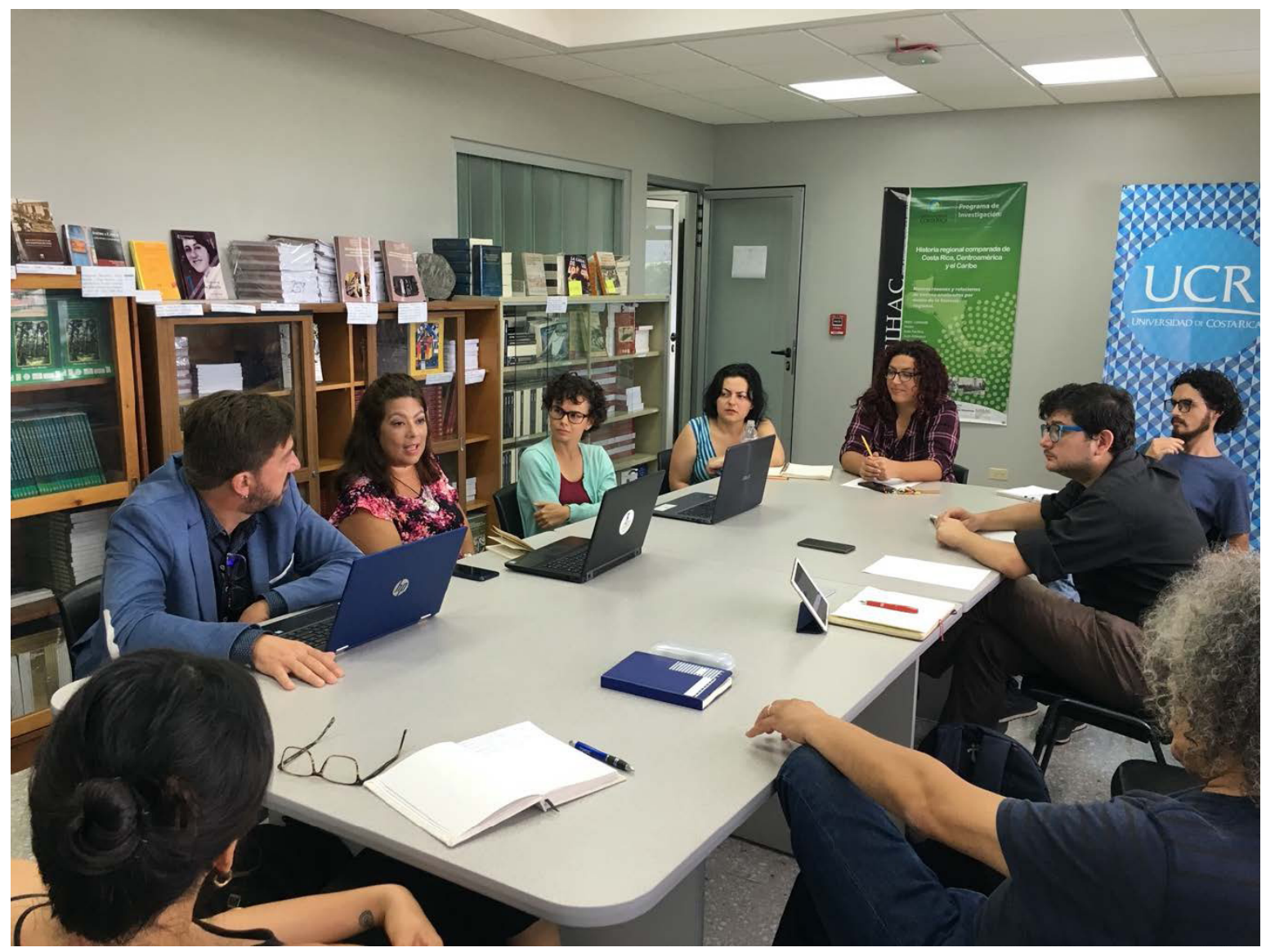

\section{Hacia unos estudios urbanos centroamericanos}

El último elemento que deseo abordar es la necesidad de establecer una red de estudios urbanos a nivel centroamericano. La participación de la Dra. Navarrete puso en la mesa de discusión la necesidad de atraer aún más investigadores que se ocupen de los fenómenos urbanos e impulsar agendas de trabajo compartidas, que puedan eventualmente servir para desarrollar síntesis analíticas y estudios comparativos en la región. Las actividades a su cargo evidenciaron que ciertos procesos urbanos, llevados a cabo en la ciudad de Tegucigalpa, compartieron similitudes con otros aplicados en Costa Rica. Claro está, resonaron también las diferencias en cuanto al alcance de las políticas urbanas y la institucionalidad que las llevaron a cabo.

La bibliografía disponible y las fuentes de información deben ser actualizadas y digitalizadas, para facilitar el acceso regional. Para esto, la creación de los repositorios es esencial en esta tarea. También es prioritario la consolidación de los nexos institucionales entre las universidades costarricenses con sus pares centroamericanas, para que proyectos conjuntos enfocados en la problemática urbana tengan sustento financiero y abran nuevos espacios de discusión o fortalezcan los existentes. Las tecnologías de la información y la comunicación, en nuestro tiempo, facilitan esta tarea y el seguimiento a las agendas de investigación se facilita enormemente.

Las I Jornadas de Estudios Urbanos fue un primer paso hacia la consecución de estas metas que ciertamente son de mediano y largo plazo. No obstante, el darle continuidad al proyecto 0 a otros similares con estos objetivos en mente, favorecerá la maduración y la visibilidad de los estudios urbanos centroamericanos y su presencia en los principales foros de discusión académica alrededor del mundo.

Quiero finalizar extendiendo un agradecimiento especial al Máster Gustavo Jiménez, con quien fue posible planificar y convocar estas Jornadas. A los y las directores y directoras de centros de investigación, laboratorios y unidades académicas de la UCR: Dr. Sergio Villena, Dra. Ana María Botey, Dra. Zuhra Sasa, Dra. Helga von Breymann, 
Dr. Anthony Goebel, Dr. Allen Cordero, Dr. David Díaz; a la Máster Marcela Otárola de la UNA; al CEM-UNAM y al Dr. Carlos Valdés y Mtro. Héctor Perdomo, a los funcionarios de la OAICE, al M.Sc. Luis Durán del LACITE, a la Oficina de Divulgación e Información de la UCR y a sus periodistas Andrea Méndez Montero y Francisco Ruiz León, al Canal 15 y a los distintos funcionarios de la UCR, estudiantes, investigadores de diversas instituciones y público en general que participaron y apoyaron este proyecto académico.

\section{Referencias Bibliográficas}

Altezor, C. (1986). Arquitectura urbana en Costa Rica: exploración histórica 1900-1950. Cartago: Editorial Tecnológica de Costa Rica.

Alvarado, A. y Jiménez, G. (2014). Acercamiento al estudio de las urbanizaciones cerradas en Costa Rica: Notas metodológicas. Revista de Ciencias Sociales, Vol. III (No 145), pp. 99-108.

Arguedas, , H. (1960). Sugestiones urbanísticas y terminal de autobuses. (Tesis de para grado de Arquitecto-Ingeniero). Universidad de Costa Rica.

Argüello, M. (1980). El desarrollo del movimiento social urbano en una región de concentración de sobrepoblación. Las luchas de los más pobres en Puntarenas. (Tesis de licenciatura en Sociología). Universidad de Costa Rica.

Bonilla, A. (2018). Circulación de saberes del urbanismo y redes transnacionales del INVU, 1954-1970. Revista Reflexiones, Vol. 97 (№ 2), pp. 86-106.

Botey, A. (2013). Los actores sociales y la construcción de las políticas de salud del Estado Liberal en Costa Rica (1850-1940). (Tesis doctoral en Historia). Universidad de Costa Rica.

Brenes, J. y Zúñiga, G. (1985). Evaluación de la ciudad de Cartago como núcleo urbano. (Tesis de licenciatura en Ingeniería civil). Universidad de Costa Rica.

Carazo, R. (1958). Breves consideraciones sobre el problema del Urbanismo. Revista de la Universidad de Costa Rica, Vol. 18, pp. 9-18.

Durán, L. (2013). Cartografías josefinas: ventas ambulantes y espacio público. Heredia: Editorial de la Universidad Nacional.

Fumero, P. (1996). Teatro público y estado en San José 1880-1914: una aproximación desde la historia social. San José: Editorial Universidad de Costa Rica.

Girot, P. (2019). Escala de acción, resiliencia urbana y equidad social. RevistaAmbientico (No 270), pp. 4-10.

Gordienko, E., (1955). Mercados centrales para provincias de Costa Rica. (Tesis de licenciatura en Ingeniería civil). Universidad de Costa Rica.

Hall, C. (1983). Costa Rica. Una interpretación geográfica con perspectiva histórica. San José: Editorial Costa Rica.

Hernández, E., Herrera, M.A. y Vargas, Fabio. (1984). Expresiones arquitectónicas y culturales en la ciudad satélite de Hatillo. (Tesis de licenciatura en Arquitectura y Antropología). Universidad de Costa Rica.

Izquierdo, C. (2016). Pobreza, condiciones de vida y subjetividad en el casco central de San José. (Tesis de maestría en Historia). Universidad de Costa Rica.

Jenkins, E. y Crespo, J. (1958). Ciudad satélite de Hatillo y Unidad Vecinal N02. Revista de la Universidad de Costa Rica, Vol. 18, pp. 43-88.

Jiménez, G. (2018). La movilidad urbana en el casco central de la ciudad de San José, Costa Rica: El caso de las motilidades de los vecinos y vecinas de Barrio Luján y Barrio Escalante. (Tesis de maestría en Movilidad, Transporte y Territorio). Universidad de Guadalajara.

Jiménez, A. (2017). "Mall"-deando la ciudad: 24 años de territorialización de los mall en Costa Rica. revistArquis. Revista de la Escuela de Arquitectura de la Universidad de Costa Rica, Vol. 6, (№ 2), pp. 71-85. 
Jiménez, F. (1985). Evaluación de las características del desarrollo urbano en el distrito central del cantón de Desamparados. (Tesis de licenciatura Ingenería civil). Universidad de Costa Rica.

Jouffe, Y. (2015). Contra el derecho a una ciudad accesible. Perversidad de una reinvindicación consensual. Revistarquis. Revista de la Escuela de Arquitectura de la Universidad de Costa Rica, Vol. I, (Nº 7), pp. 34-44.

Lungo, M. (2004). Centroamérica, Globalización, Tratado de Libre Comercio con los Estados Unidos y Metropolización transnacional. Cadernos PROLAM/USP, Año 3, (Vol. 2), pp. 7-28.

Lungo, M. (2015). Globalización grandes proyectos y privatización de la gestión urbana. Revista Urbano, Vol. 8, (№ 11), pp. 49-58.

Malavassi, R. (2014). La vivienda de madera de los barrios Luján-El Cerrito y Barrio Keith (1910-1955). Un análisis histórico de la imagen urbana y la arquitectura habitacional. (Tesis de maestría en Historia). Universidad de Costa Rica.

Martínez, T. (2009). Nuevas tendencias de nuevas polis. Del encuentro al flujo. Cartago: Editorial Tecnológica de Costa Rica.

Molina, W. (2019). La gentrificación en Costa Rica: Elementos para su estudio y comprensión. Revista de Ciencias Sociales, Vol. II (№164), pp. 87-107.

Montoya, A. (1976). El problema de la vivienda y el urbanismo en Costa Rica. (Tesis de licenciatura en Sociología). Universidad de Costa Rica.

Morales, M. (Ed.). (1976). El proceso de metropolización en Costa Rica y América Latina. San José: Escuela de Historia y Geografía, IGN, MOPT.

Navarrete, D. (2012). Tegucigalpa. Política y urbanismo, 1578-1949. Tegucigalpa: Instituto Hondureño de Antropología e Historia.

Navarrete, D. (2018). Tegucigalpa, laboratoire urbain des modernités au Honduras XIXe et XXe siècles. (Thèse de doctorat en Études Urbains). Écoles des hautes études en sciences sociales.

Palmer, S. (1996). Prolegómenos para toda historia futura de San José, Costa Rica. Mesoamérica, Vol. 17 (№31), pp. 181-213.

Pérez, S., y Pujol, R. (Ed.). (2003). Desafíos de los centros de las ciudades mesoamericanas. Los casos de tres metrópolis. San José: FLACSO.

Picado, C. (2019). Resiliencia humana en la agenda urbana de Costa Rica. Revista Ambientico ( $\left.{ }^{\circ} 270\right)$, pp. 11-17.

Ruiz, L. F. (2019, Julio 11). Una ciudad es una comunidad política. Recuperado de: https://www.ucr.ac.cr/noticias/2019/07/11/una-ciudad-es-una-comunidad-politica. html.

Quesada, F. (2004). En el Barrio Amón: arquitectura, familia y sociabilidad del primer residencial de la élite urbana de San José, 1900-1935. San José: Editorial Universidad de Costa Rica.

Quesada, F. (2011). La modernización entre cafetales: San José, Costa Rica, 18901930. San José: Editorial Universidad de Costa Rica.

Sánchez A., Paniagua, L., Brenes, M. (2012). San José, una ciudad en perpetua transformación. Reflexiones sobre el espacio. Revista de Ciencias Sociales, Vol. III ( $\left.\mathrm{N}^{0} 137\right)$.

Sánchez, N., Umaña, C. (1982). San José, imagen y estructura urbana. (Tesis de licenciatura en Arquitectura). Universidad de Costa Rica.

Séguin, A. y Negron, P. (Ed.) (2006). La segregación socio-espacial urbana: Una mirada sobre Puebla, Puerto España, San José y San Salvador. San José: FLACSO.

Van Lidth de Jeude, M. y Schütte, O. (2013). El Círculo Vicioso de la Fragmentación Espacial y Segregación Social en la Gran Área Metropolitana de Costa Rica. revistArquis. Revista de la Escuela de Arquitectura de la Universidad de Costa Rica, Vol. II (N 3), pp. 22-43. 
Vargas, E. (1958). Principios teóricos sobre urbanismo. Revista de la Universidad de Costa Rica, Vol. 18, pp. 19-42.

Woodbridge, R. (1988). Historia de la Arquitectura en Costa Rica. Cartago: Editorial Tecnológica de Costa Rica. 